\title{
Ambulatory monitoring for obstructive sleep apnea: It's not the device that matters, but how the data are used
}

\author{
Frank Ryan MB FRCPC
}

$\mathrm{M}$ en and women with obstructive sleep apnea (OSA) may differ in their symptomatology and polysomnographic manifestations, which leads to the potential for underdiagnosis in women. In the September/October issue of the Canadian Respiratory Journal, Gjevre et al (1) reported that an unattended, in-home diagnostic sleep test was useful in identifying OSA in women. Their study is important because ambulatory monitoring in the diagnosis of OSA remains to be broadly validated and, to date, most studies have disproportionately recruited men.

OSA is a common condition that affects $4 \%$ to $5 \%$ of the adult population. Facility-based polysomnography (PSG) has traditionally been used to diagnose OSA and to establish optimal treatment, and continues to be the recommended approach when readily available. The main argument for PSG has been its ability to monitor sleep and the precision with which it identifies and classifies respiratory events. Unfortunately, access to facility-based PSG is limited because of its technical complexity and expense. It is estimated that $80 \%$ to $90 \%$ of patients with OSA remain undiagnosed (2).

The continuing recommendations to use PSG in the initial assessment and management of patients with suspected OSA raises some important issues in relation to diagnosis, treatment and access to care. First, it is arguable whether the degree of precision provided by PSG is warranted for the majority of patients. Knowing the precise number of obstructed respiratory events during sleep has limited clinical utility, particularly because the diagnosis of OSA-hypopnea syndrome (OSAHS) requires additional clinical information such as the presence of symptoms. Moreover, there is no sound pathophysiological rationale for selecting a particular cut-off value of the apnea-hypopnea index (AHI) as being diagnostic of OSA. The correlation between the severity of OSA as measured by AHI during PSG and either symptoms or response to treatment is poor. Furthermore, the majority of patients in the community have uncomplicated OSA with either no significant or easily identifiable comorbidities, or other factors, that could lead to diagnostic confusion.

Second, there is a currently available, highly effective treatment for OSA that works regardless of the severity or the cause of the condition. Continuous positive airway pressure (CPAP), applied by a closefitting mask over the nose and/or mouth, provides a pneumatic splint for the upper airway, propping it open and preventing it from occluding during sleep. Newer CPAP machines are able to deliver a level of positive airway pressure that is autotitratable to the patient's needs, enabling this treatment to be initiated in the patient's home and a therapeutic trial undertaken before committing to the purchase of expensive equipment. CPAP is safe and has a very low likelihood of causing harm if administered unnecessarily to a patient in whom OSA has been diagnosed in error. Furthermore, poor tolerance of CPAP or persistent symptoms despite CPAP are suggestive of an incorrect or incomplete diagnosis.

Regarding access to PSG, the demand has far outstripped the available resources. Budgetary constraints on the health care system in Canada and elsewhere make it unlikely that we will see any significant expansion of facility-based PSG resources in the future.
Increasing recognition of these factors has prompted a quest for cheaper and more accessible alternatives for the initial management of patients with suspected OSA. In 2007, the Centre for Medicare and Medicaid Services (CMS) in the United States conducted a technology assessment of ambulatory monitoring in the diagnosis of OSA, which included a systematic review of the literature and a modelling exercise $(3,4)$. The primary question addressed by the CMS was whether diagnostic strategies other than facility-based PSG could accurately identify patients with OSA who would benefit from CPAP treatment. They concluded that there was sufficient evidence that, in appropriately selected patients, some home sleep test monitors can identify a significant proportion of patients with OSA who will respond to CPAP, and will exclude a significant proportion of those who will not. The CMS also concluded that home sleep testing improves health outcomes in patients who have OSA and receive appropriate treatment. The CMS review listed several caveats that could limit the broader application of their conclusions. In general, the studies they reviewed involved patients who were younger, predominantly male and obese, with a high likelihood of OSAHS, absence of comorbidities, and with no previous surgery for snoring or OSAHS. In 2011, the Canadian Thoracic Society (CTS) issued updated guidelines on portable monitoring in OSA following a systematic review that focused only on other systematic reviews and randomized controlled trials (5). These guidelines state that, while facility-based PSG remains the accepted standard for the diagnosis of OSA and the method of choice when readily available, portable monitoring studies can be used to confirm OSAHS and institute appropriate treatment in patients who have a moderate-to-high pretest probability of OSA, and when these studies are integrated into a package of care that includes appropriate expertise (physicians and other health care workers) and the back-up availability of PSG. The CTS guidelines also include caveats regarding the use of portable monitoring in patients with comorbid diseases that may confound the diagnosis and draw attention to the limitations of oximetry in differentiating between different types of sleep disordered breathing.

Clearly, over the past few years, new evidence has prompted expert opinion to move in favour of using portable monitoring in appropriately selected patients with OSA while acknowledging some important caveats. Both the CMS and CTS documents, however, pointed to the need for broader validation of portable monitoring in the assessment of patients with suspected OSA before its widespread adoption.

One of the limitations in the current literature on portable monitoring in OSA has been the low proportion of women enrolled in studies to date. This is perhaps not surprising because there is a higher prevalence of OSA in men. Women with OSA may have different symptomatology and lower AHIs, leading to the potential for underdiagnosis. In the September/October issue of the Journal, Gjevre et al (1) addressed this limitation by examining the utility of a portable monitoring device in a group of women with suspected OSA. Consecutive women between 21 and 70 years of age scheduled for routine PSG testing for evaluation of clinically suspected OSA were invited to participate. Exclusion criteria were a strong suspicion of

University of British Columbia, The Lung Centre, Vancouver, British Columbia

Correspondence and reprints: Dr Frank Ryan, University of British Columbia, The Lung Centre, Diamond Health Care Centre, 7th Floor,

2775 Laurel Street, Vancouver, British Columbia V5Z 1M9. Telephone 604-875-4122, fax 604-875-4695, e-mail frank.ryan@vch.ca 
another primary sleep disorder, regular shiftwork in the previous six months, pregnancy or significant medical comorbidities. Of 96 patients who met the study criteria, 47 consented to participate. Unattended, in-home monitoring was performed in random sequence one week before or after PSG testing using the Embletta (Embletta, USA) device, which monitors oxygen saturation, heart rate, respiratory movements, airflow, snoring and body position. The main variable of interest from the in-home monitoring was AHI. Using PSG as the gold standard, they calculated sensitivities, specificities, likelihood ratios and predictive values for different cut-off levels of AHI derived from the portable device. An AHI $>10$ produced the highest level of agreement with PSG. As the AHI cut-off increased from 5 to 30, sensitivity decreased and specificity increased. An AHI of $>15$ had a positive predictive value of greater than $95 \%$ for OSA but a negative predictive value of only $54 \%$ or less. The ambulatory device was less useful at distinguishing the degree of severity of OSA. Overall, the diagnostic utility of the Embletta device in this study, which indicated moderate decreases or increases in the likelihood of OSA based on AHI cut-offs of $<5$ and $>15$, respectively, was similar to that in other reports of ambulatory devices in patients with suspected OSA (3).

A strength of the study by Gjevre et al was that it compared PSG and ambulatory devices on different nights and in different settings (laboratory versus home). This study design represents a more realistic test of the ambulatory device as opposed to a simultaneous comparison in the laboratory. Although the investigators stated that their data were based on patients at high risk for OSA, this was not an explicit selection criterion for their study. This is an important point because the utility of a test is heavily influenced by the prior probability of disease. In a randomized validation study, Mulgrew et al (6) showed that, in a sleep clinic setting where the prevalence of moderate-tosevere OSA was 50\%, an ambulatory device used in conjunction with a clinical prediction algorithm could identify patients with a $>95 \%$ pretest probability of moderate-to-severe OSA. When these patients were treated with CPAP at a pressure initially determined by autotitration in the home, outcomes after three months of treatment were not inferior to patients managed using facility-based PSG. This suggests that the utility of ambulatory monitoring in clinical practice may depend more on how the results are incorporated into a comprehensive diagnostic and management algorithm than on the precise technical or diagnostic performance characteristics of the device itself. There is ample evidence that ambulatory devices correlate reasonably well with PSG, which the study by Gjevre et al also confirmed. The challenge now is to learn how to use these devices optimally. The probability of disease can be expressed as a point on a continuum between zero and one. For example, a patient who snores but is not sleepy and has no other symptoms or clinical features suspicious for OSA, has a very low pretest probability of disease. A negative ambulatory test in such a patient will indicate a low likelihood for OSA. Conversely, a patient with a high score on a clinical prediction rule for OSA has a high pretest probability, and a positive ambulatory study will indicate a high likelihood for OSA. Within the context of a structured sleep program, these patients can be safely managed without the need for PSG. The difficulty with an ambulatory approach based on estimates of disease probability arises from the intermediate or indeterminate group of patients lying between the two ends of the probability spectrum. We need to define the limits of both low and high probability categories of patients for whom an ambulatory approach can be safely used. To address this challenge, broader validation and impact analysis of ambulatory monitoring in the management of OSA is needed. This will require larger randomized clinical trials using less restrictive selection criteria encompassing a broader range of OSA severity and the inclusion of patients with comorbidities (7). More studies should be conducted in settings outside of the carefully controlled environs of the sleep centre, such as in the community or in general practice clinics, where the prevalence of OSA is lower and the range of expertise in sleep disorders is more heterogeneous (8). Better clinical prediction rules are needed to more accurately estimate pretest probability. Finally, cost-effectiveness studies are needed to confirm that ambulatory approaches are indeed cheaper. The study by Gjevre et al is a step along the way because it provides reassurance regarding the utility of ambulatory monitoring in women with suspected OSA.

In the future, rational clinical decision making by appropriately trained clinicians using validated clinical prediction rules, together with increasingly sophisticated home sleep monitoring and autotitrating positive airway pressure devices, will likely be the mainstay for the initial diagnosis and management of OSA. Facility-based PSG will be reserved for special circumstances such as when there is considerable diagnostic uncertainty, or when treatment responses are unfavourable or uncertain, when technical issues preclude home sleep testing or when medicolegal considerations such as patients with safety critical occupations mandate a definitive diagnostic test.

\section{REFERENCES}

1. Gjevre JA, Taylor-Gjevre RM, Skomro R, et al. Comparison of polysomnographic and portable monitoring assessments of obstructive sleep apnea in Saskatchewan women.

Can Respir J 2011;18:271-4.

2. Young T, Evans L, Finn L, et al. Estimation of the clinically diagnosed proportion of sleep apnea syndrome in middle-aged men and women. Sleep 1997;20:705-6.

3. Trikalinos T, Ip S, Raman G, et al. Home diagnosis of obstructive sleep apnea hypopnea syndrome. Agency for Healthcare Research and Quality, Bethesda: Department of Health and Human Services, USA, 2007.

4. Trikalinos TA LJ. Obstructive sleep apnea hypopnea syndrome: Modeling different diagnostic strategies. Agency for Healthcare Research and Quality, Bethesda: Department of Health and Human Services, USA, 2007.

5. Fleetham J, Ayas N, Bradley D, et al. Canadian Thoracic Society 2011 guideline update: Diagnosis and treatment of sleep disordered breathing. Can Respir J 2011;18:25-47.

6. Mulgrew AT, Fox N, Ayas NT, et al. Diagnosis and initial management of obstructive sleep apnea without polysomnography: A randomized validation study. Ann Intern Med 2007;146:157-66.

7. Kuna ST, Gurubhagavatula I, Maislin G, et al. Noninferiority of functional outcome in ambulatory management of obstructive sleep apnea Am J Respir Crit Care Med 2011;183:1238-44.

8. Antic NA, Buchan C, Esterman A, et al. A randomized controlled trial of nurse-led care for symptomatic moderate-severe obstructive sleep apnea. Am J Respir Crit Care Med 2009;179:501-8. 


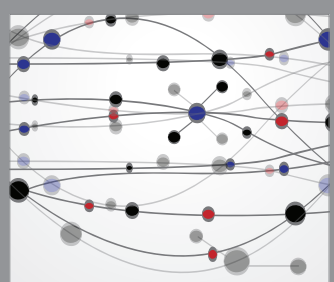

The Scientific World Journal
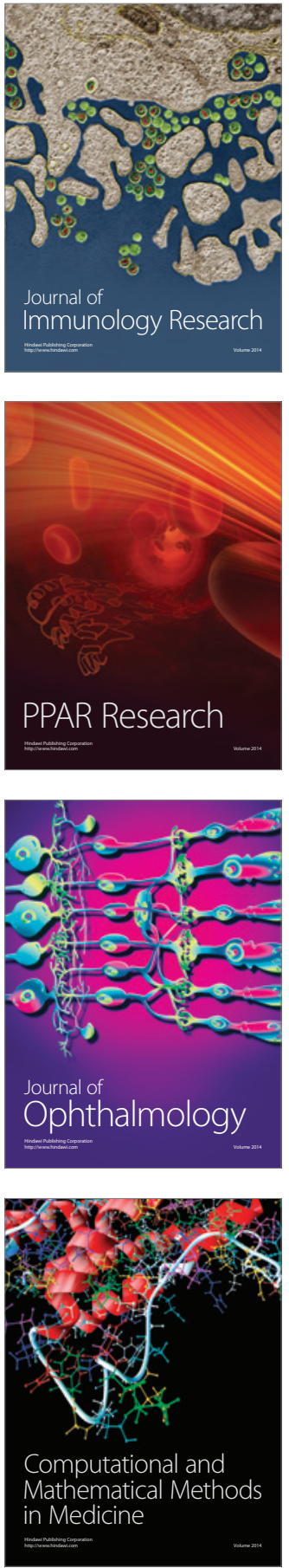

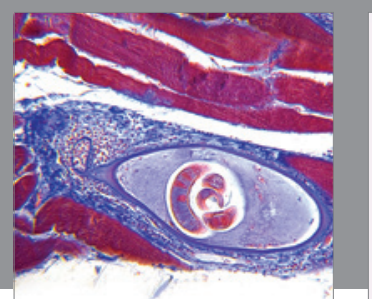

Gastroenterology Research and Practice

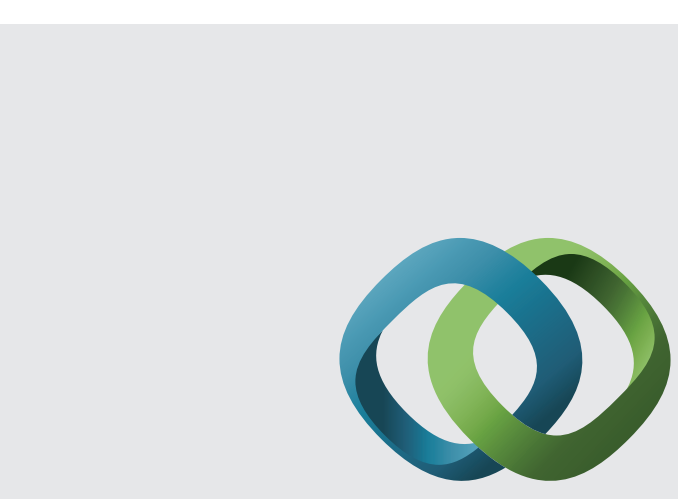

\section{Hindawi}

Submit your manuscripts at

http://www.hindawi.com
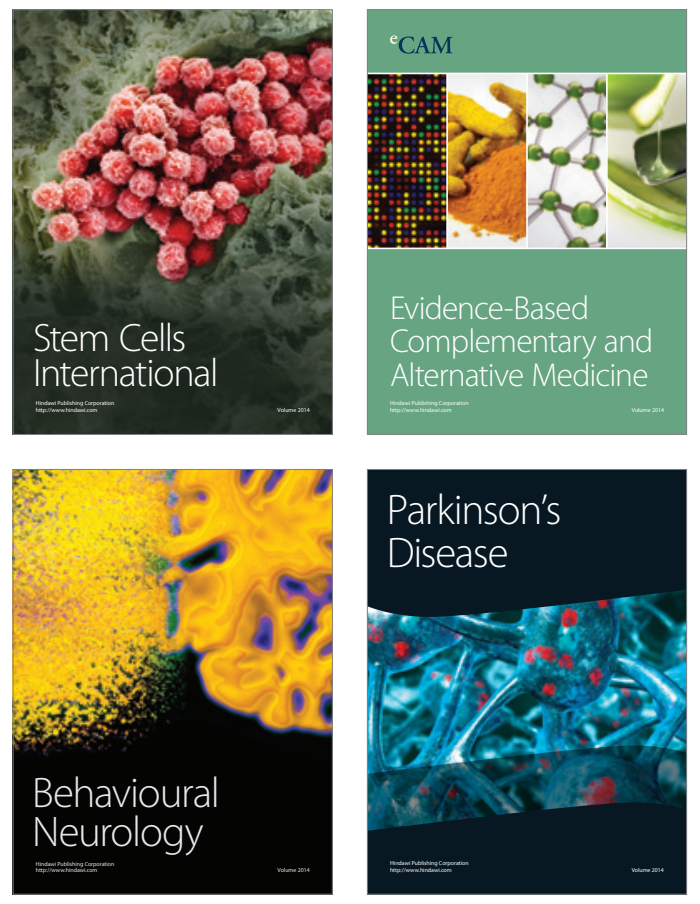
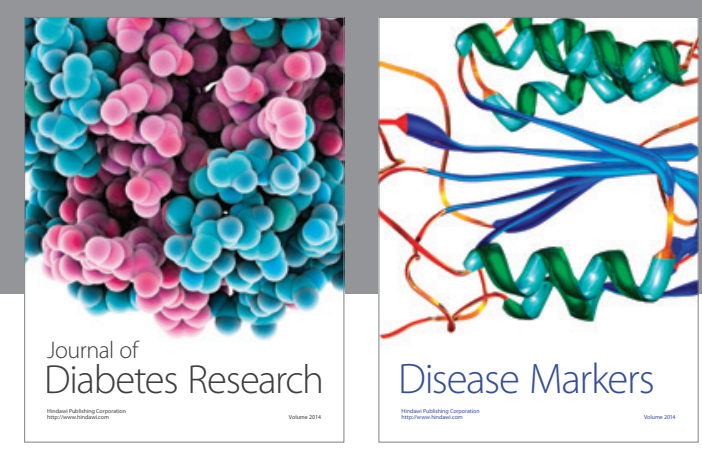

Disease Markers
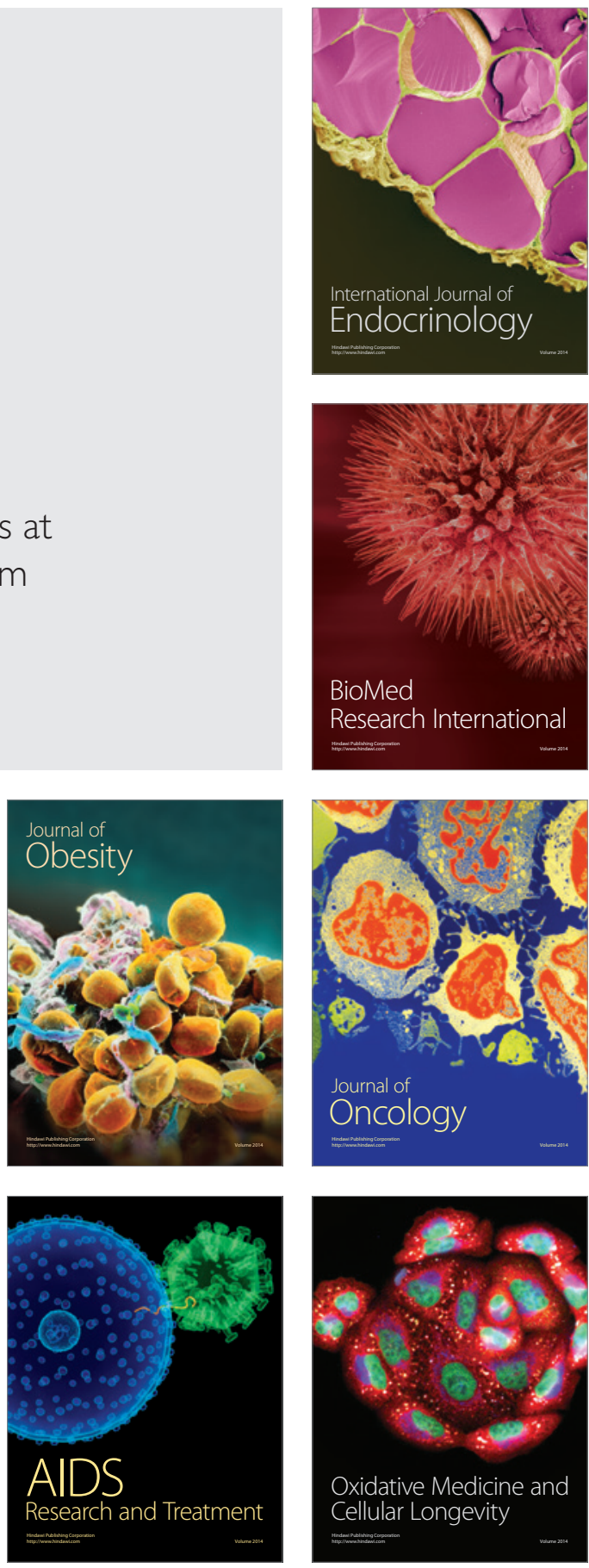